\title{
Vom Bleistift zum Prozessor: Wandel der Modelle in der Siedlungsentwässerung
}

Zusammenfassung: Die Entwicklung von numerischen Methoden führte in den letzten Jahren zu einem radikalen Wandel in der Dimensionierung von Anlagen der Siedlungsentwässerung. Waren bislang Berechnungsformeln und empirische Beziehungen vorherrschend, so wird heute in den Richtlinien der Einsatz von Software Werkzeugen als Stand der Technik vorgeschrieben. Dem erhöhten Aufwand in der Projektierung steht der Vorteil optimierter und damit kostengünstiger Lösungen gegenüber.

From the pencil to the processor: Change in the modelling of urban sewerage systems

Summary: The development of numerical models has led to a radical change in the technical design of urban sewerage facilities. Whereas calculation formulas and empirical relations prevailed until recently, directives now dictate the use of software as state-of-the-art tools. The increased design cost is outweighed by the advantage of optimised and thus lowercost solutions.

\section{Einleitung}

Anlagen und Methoden der Siedlungsentwässerung müssen zwei grundsätzliche Aufgabenstellungen erfüllen, das sind erstens "Schutz des Menschen vor der Natur“ und zweitens „Schutz der Natur vor dem Menschen“. Während die Entwässerungssysteme unter Trockenwetterbedingungen keine Probleme aufwerfen, stellen Starkniederschläge für beide Aufgaben die maßgeb- lichen Anforderungen dar. Die erste Aufgabenstellung umfasst daher die Konzeption und Dimensionierung des Kanalsystems in einer Art und Weise, dass Überlastungen des Systems (d.h. Überflutungen der urbanen Flächen) nur mehr sehr selten (z. B. weniger als einmal pro Jahr) auftreten. Eine vollständige Vermeidung ist - analog zum Hochwasserschutz - weder technisch möglich noch ökonomisch sinnvoll.

Für den Schutz der Gewässer ist die Siedlungsentwässerung dergestalt zu konzeptionieren, dass bei Regenwetter eine möglichst geringe Stofffracht über die Mischwasserentlastung in die Gewässer geleitet wird, bzw. ein möglichst hoher Prozentsatz an der Kläranlage behandelt wird. Die vollständige Behandlung des Abflusses unter Trockenwetterbedingungen an der Kläranlage stellt kein Problem der Siedlungsentwässerung dar und wird vorausgesetzt. Die beiden primären Ziele der Bemessung lauten daher:

- Bemessung des Kanalsystems im Hinblick auf Überflutung

- Bemessung des Kanalsystems im Hinblick auf entlastete Stofffrachten

Für diese beiden Dimensionierungsaufgaben wurden historisch empirische Beziehungen und einfache Berechnungsformeln verwendet, es handelte sich also um so genannte „Papier und Bleistift" Berechnungen. In den letzten beiden Jahrzehnten wurden aber vermehrt numerische Modelle verfügbar, die eine detaillierte Simulation der Dynamik der Vorgänge bei Regenwetter ermöglichen. Dies führte auch zu einem radikalen Wandel in den Richtlinien, indem seit kurzem die numerische Simulation als Stand der Technik im Entwurf von Kanalisationssystemen vor- geschrieben wird. Der vorliegende Artikel beschreibt den Wandel der Modelle und zeigt den Einsatz modernen Software Werkzeuge an einem konkreten Beispiel.

\section{Richtlinien, Modelle und Software}

\subsection{Historische Entwicklung von Modellen und Software}

Durch Arbeiten von Mulvaney (1851) in Irland und Kuichling (1889) in der USA stand bereits zu Beginn des modernen Kanalbaus mit dem Fließzeitverfahren eine Methode zur quantitativen Berechnung des Abflusses unter Regenwetterbedingungen zur Verfügung. Dieser klassische Ansatz der Listenrechnung wird - mit Modifikationen - bis heute im Entwurf von Kanalisationen verwendet, allerdings in den letzten 20 Jahren zunehmend ersetzt durch numerische Modelle.

Die Entwicklung von numerischen Berechnungsmethoden wurde maßgeblich von der US-EPA beeinflusst, die bereits 1969 ein Konsortium unter Führung von Metcalf and Eddy mit der Erstellung der Software SWMM (Stormwater Management Model) beauftragte. Seit der ersten Version im Jahre 1971 wurde dieses Berechnungswerkzeug bis heute kontinuierlich weiterentwickelt. Mit SWMM 5 steht ein hydrodynamisches Berechnungsmodell zur Simulation von Abflüssen und Stofffrachten in Kanalsystemen zur Verfügung, das aufgrund des Status als „public domain software" eine sehr weite Verbreitung aufweist.

Ausgehend von diesem Modell wurden seit den 80er Jahren auch in Europa verschiedene Modelle und Softwareprodukte

\begin{tabular}{|c|c|c|c|c|c|c|c|c|c|c|}
\hline Nr. & $\begin{array}{l}A_{E, k} \\
\text { (ha) }\end{array}$ & $\begin{array}{c}\Psi \\
(-)\end{array}$ & $\begin{array}{l}A_{\text {red }} \\
\text { (ha) }\end{array}$ & $\underset{\text { (ha) }}{\boldsymbol{\Sigma} A_{\text {red }}}$ & $\begin{array}{c}\mathrm{L} \\
(\mathrm{m})\end{array}$ & $\begin{array}{c}\mathbf{v} \\
(\mathrm{m} / \mathrm{s})\end{array}$ & $\underset{(\min )}{\mathbf{t}}$ & $\underset{(\min )}{\Sigma t}$ & $\underset{(-)}{\varphi}$ & $\begin{array}{c}a_{R} \\
(1 / s)\end{array}$ \\
\hline S7 & 8,7 & 0,3 & 2,6 & 2,6 & 212 & 1,0 & 3,5 & 8,5 & 1,64 & 640 \\
\hline S6 & 11,0 & 0,3 & 3,3 & 3,3 & 278 & 1,0 & 4,6 & 9,6 & 1,64 & 812 \\
\hline S5 & 5,2 & 0,5 & 2,6 & 8,5 & 192 & 1,0 & 3,2 & 12,8 & 1,43 & 1823 \\
\hline S4 & 6,2 & 0,5 & 3,1 & 11,6 & 191 & 1,0 & 3,2 & 16,0 & 1,25 & 2175 \\
\hline
\end{tabular}


für die dynamische Simulation des Abflusses entwickelt. Bekannt und kommerziell erfolgreich sind hier vor allem Hystem-Extran und Kanal++ aus Deutschland, Mouse bzw. MikeUrban aus Dänemark und InfoWorks aus England.

Im Vergleich zur Abflussberechnung wurde die Frage des Gewässerschutzes im Deutschsprachigen Raum historisch direkt an die Bemessung von Mischwasserentlastungbecken geknüpft. D.h. es wurden empirische Regeln für die Dimensionierung der Becken entwickelt, welche vereinfacht das Systemverhalten und die Redundanz des Vorfluters berücksichtigen. Diese simplen Bemessungsformeln wurden nur langsam durch numerische Berechnungsmethoden ersetzt - in Österreich z.B. erst mit der Neufassung des Regelblattes 19 im Jahre 2007.

Die numerische Berechnung der Stofffrachten bedingt die kontinuierliche Simulation des Geschehens über einen relevanten Zeitraum von ca. 10 Jahren. Obwohl hierzu grundsätzlich auch die vorhin erwähnten hydrodynamischen Modelle in der Lage sind, wurden wegen der geringeren Rechendauer einfachere hydrologische Modelle entwickelt. Erste Softwareprodukte entstanden meist in Zusammenhang mit Dissertationen z. B. entwickelte Paulsen (1987) das Werkzeug KOSIM (Uni Hannover) und Johansen (1985) SAMBA (TU Dänemark). In den folgenden Jahren wurden weitere Software Werkzeuge entwickelt - meist auch in direktem Zusammenhang mit den Landesspezifischen Regelwerken: z. B. REBEKA in der Schweiz (Rauch et al., 2002) und KAREN für Österreich (Rauch und Kinzel, 2007).

\subsection{Regelblatt 11}

Das Regelblatt 11 wurde vom Österreichischen Wasser- und Abfallwirtschaftsverband 2009 veröffentlicht und befasst sich mit der hydraulischen Berechnung von Kanalsystemen. Als wesentliche Neuerung ist hier das traditionelle Fließzeitverfahren nur mehr für einfache Systeme und Neuplanungen zulässig. Übliche komplexe Entwässerungssysteme sind mittels numerischer Simulation nachzuweisen, wobei auch das unbedingt notwendige Ausmaß der Kalibrierung spezifiziert wird. Obwohl mit diesem Regelblatt die Verwendung von Software als Stand der Technik fixiert wird, erfolgt keine konkrete Vorgabe hinsichtlich eines speziellen Produktes. Nachzuweisen sind Überstaubzw. Überflutungshäufigkeiten nach den Vorgaben der ÖNORM EN 752, d.h. in Ab-

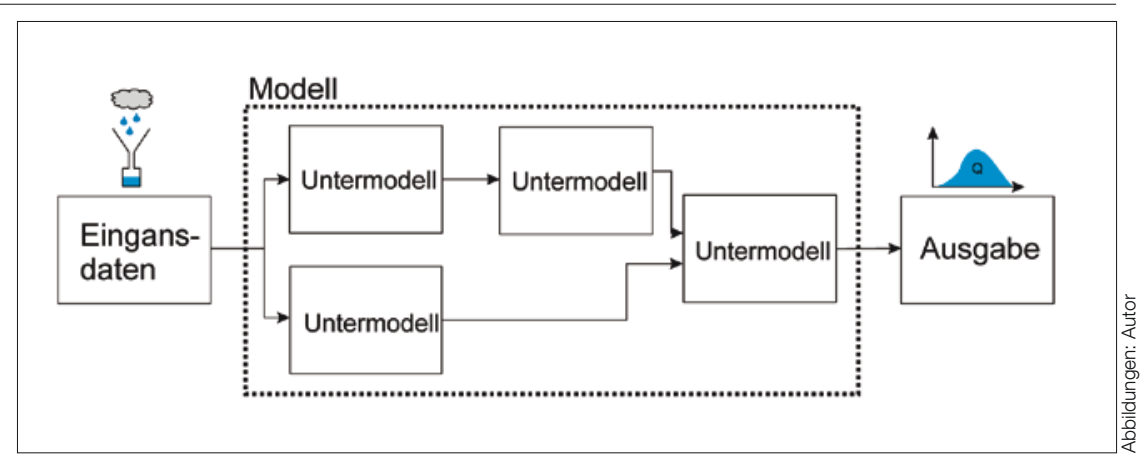

Abb. 1: Schema eines dynamischen Modells.

hängigkeit der Nutzung der Bereiche. Z. B. können ländliche Gebiete rechnerisch 1 mal in 2 Jahren überstaut werden, während Stadtzentren nur alle 5 Jahre überstaut werden sollen. Für die Berechnung sind entweder Modellregen (empfohlen wird der Typ Euler II) zu verwenden oder eine Starkregenserie die aus mindestens 10 Jahren Niederschlagsaufzeichnungen extrahiert wird.

\subsection{Regelblatt 19}

2007 wurde vom Österreichischen Wasser- und Abfallwirtschaftsverband eine Neufassung des Regelblattes 19 veröffentlicht welche sich mit der Bemessung der Mischwasserbehandlung in Kanalsystemen befasst. Wie beim Regelblatt 11 wird auch hier ein radikaler Wechsel in der Bemessung vorgeschlagen, indem die kontinuierliche Simulation des Entwässerungssystems als Stand der Technik festgelegt wird. Mit Hilfe der numerischen Modellierung wird derjenige Anteil des Niederschlagsabflusses (bzw. der im Regenwetterfall abfließenden Stofffrachten), der zur Kläranlage geleitet wird, berechnet. Dieser Anteil muss einem vorgegeben Wirkungsgrad entsprechen. Der Wirkungsgrad selber ist wiederum von der Größe des betrachteten Einzugsgebietes und dem Niederschlagsgeschehen abhängig die Werte liegen zwischen $40 \%$ und $60 \%$ für gelöste Stoffe bzw. zwischen $55 \%$ und $75 \%$ für abfiltrierbare Stoffe. Der Vorteil dieser neuen Methodik liegt darin, dass nicht mehr singuläre Maßnahmen behandelt werden, sondern das Verhalten des Gesamtsystems berücksichtigt wird. Zudem sind nicht mehr nur Speicherbecken als Behandlungsmaßnahmen zulässig, sondern auch Regenwasserversickerungen, Steuerungsmaßnahmen usw. Für die Berechnung sind kontinuierliche Regenaufzeichungen über mindestens 10 Jahre notwendig. Diese können entweder über eigene Messungen oder hydrographische
Dienste bezogen werden oder im Zusammenhang mit dem ÖWAV-Leitfaden „Niederschlagsdaten zur Anwendung der ÖWAV-Regelblätter 11 und 19“ als DVD „NIEDA“ beim Österreichischen Normungsinstitut (www.on-norm.at). Auch in diesem Regelblatt werden nicht spezielle Softwareprodukte als Stand der Technik vorgegeben. In Österreich werden derzeit hauptsächlich KOSIM (ITWH) und KAREN (hydro-IT) verwendet.

\section{Modellierung}

Ein Modell ist eine schematische, mathematische Beschreibung von physikalischen Zusammenhängen mit dem Zweck komplexe Vorgänge fassbar zu machen, Zusammenhänge zu erklären und Vorhersagen zu machen. In der Siedlungsentwässerung dienen Modelle als Planungswerkzeug für die Bemessung und den Nachweis der Leistungsfähigkeit unterschiedlicher Infrastruktureinrichtungen wie beispielsweise zur Dimensionierung von Kanalisationssystemen, Mischwasserüberlaufbauwerken aber auch von Kläranlagen, Infiltrationsanlagen usw. Oft wird dabei ein bestimmter Vorgang durch ein Untermodell beschrieben und mehrere Untermodelle werden zu einem Modell zusammengefasst.

Zwei Begriffe, die oft als Synonym verwendet werden, dabei aber eigentlich zwei völlig unterschiedliche Dinge darstellen, sind "Software“ und „Modell“. Diese Unterscheidung ist deshalb von Bedeutung, da es in der Siedlungsentwässerung eine Vielzahl unterschiedlicher Softwareprodukte gibt, die aber meist sehr ähnliche Berechnungsalgorithmen, also Modelle, im Hintergrund haben. Die Beschäftigung mit dem Modell selbst, seinen Modellparameteren, den implizit getroffenen Annahmen und Vereinfachungen sind für den Anwender von größter Bedeutung um die Grenzen des Modells, die notwendigen Datengrundlagen usw., zu kennen. 

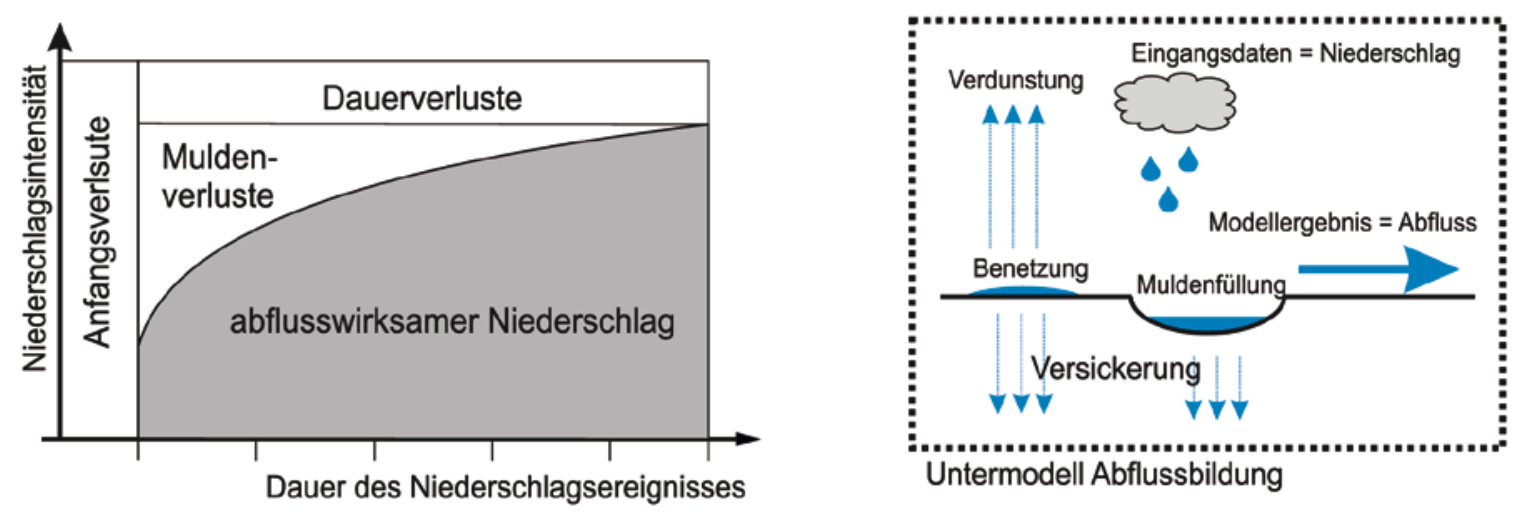

Abb. 2: Schematische Darstellung der Abflussbildung nach der Grenzwertmethode.

\subsection{Modellkonzepte in der Siedlungsentwässerung}

Niederschlag ist die treibende Kraft in allen Simulationsmodellen der Siedlungsentwässerung. Ausgehend von gemessenen oder statistisch generierten Niederschlagsdaten in hoher zeitlicher Auflösung von z.B. 5 Minuten (d.h. alle 5 Minuten wird ein Wert aufgezeichnet) wird der Regenabfluss aus einem Einzugsgebiet berechnet (Niederschlag-Abfluss Modellierung) und in weiterer Folge der Verlauf der Welle in den Kanalrohren (Transportprozesse). In den Rohren können sich Wellen durch Zusammenflüsse aus verschiedenen Einzugsgebieten überlagern und damit ansteigen oder durch eine Dämpfung abschwächen. Welche Effekte hier berücksichtigt werden, hängt von den verwendeten Transportgleichungen ab.

In der Niederschlags/Abfluss Berechnung werden üblicherweise die beiden Prozesse Abflussbildung (Wie viel des gefallenen Niederschlags kommt zum Abfluss?) und Abflusskonzentration (Welche Form hat die Abflusswelle aus dem Einzugsgebiet?) getrennt gesehen. In der Berechnung der Transportprozesse (Wie fließt die Welle in den Haltungen ab?) wird üblicherweise zwischen zwei Berechnungsansätzen „hydrodynamisches“ und „hydrologisches“ Modell unterschieden. Beide letztgenannten Ansätze unterscheiden sich nur darin wie detailliert die Transportvorgänge im Kanal berechnet werden. Die verschiedenen Untermodelle (Abflussbildung, Abflusskonzentration, Kanaltransport) werden miteinander kombiniert, wobei durchaus unterschiedliche Ansätze (komplex und einfach) miteinander verknüpft werden können.

Werden Stofffrachten berücksichtigt, müssen natürlich zwei weitere Prozesse beachtet werden. Dies ist erstens die Entstehung der Verschmutzung aus den drei Quellen Trockenwetterabfluss, Niederschlag und mobilisierte Stoffe im Kanal und zweitens der Transport der Stoffe in der fließenden Welle. Der zweite Prozess kann für gelöste Stoffe mit der bekannten Advektions/Dispersiongleichung - zumindest theoretisch - gut bestimmt werden, während der Transport von partikulären Stoffen bis heute ein Problem darstellt.

\subsubsection{Abflussbildung}

Abflussbildung ist jener Prozess, in welchem der effektive Niederschlag, der letztendlich zum Oberflächenabfluss beiträgt aus dem gemessenen Niederschlag berechnet wird. Dabei wird in einem Einzugsgebiet nicht der gesamte gemessene Niederschlag zum Abfluss kommen, sondern bestimmte Teile werden im Gebiet selbst zurückgehalten. Dieser Gebietsrückhalt ergibt sich aus den während des Niederschlagsereignisses auftretenden Verlusten (jene Anteile des Regenwassers, die nicht oberflächlich abfließen). Der summierte abflusswirksame Niederschlag wird daher immer kleiner sein als das Volumen des real gefallenen Niederschlags.

Trockene Oberflächen werden zu Beginn eines Niederschlagsereignisses benetzt. Mit der Benetzung setzt auch die Verdunstung ein. Auf durchlässigen Flächen beginnt nach der Benetzung die Versickerung. Der Muldenrückhalt bezeichnet das Auffüllen von Bodenunebenheiten mit Niederschlagswasser. Die Dauerverluste schließlich stehen zusammenfassend für eine Vielzahl an Effekten wie Verwehungen, Ableitungen, Versickerung etc., die dazu führen, dass auch bei länger anhaltenden Ereignissen nicht der gesamte Niederschlag abfließt. Diese Ver- lustprozesse treten generell bei allen Arten von Oberflächen auf, allerdings mit unterschiedlicher Relevanz je nach Oberflächentyp. Die Infiltration ist beispielsweise auf befestigten Flächen vernachlässigbar. Andererseits ist auf unbefestigten Flächen die Infiltration bedeutend, weshalb der um Größenordnungen kleinere Muldenverlust dort eine eher untergeordnete Rolle spielt. Verschiedenste Untersuchungen zeigen, dass auch simple Modelle - die diese Prozesse stark vereinfacht abbilden - das Abflussgeschehen gut wiedergeben.

Für eine numerische Simulation sind also detaillierte Informationen über den Befestigungsgrad des Einzugsgebietes notwendig. Für die Berechnung wird dann die Niederschlags/Abflussberechnung mit unterschiedlichen Modellen getrennt für befestigte und unbefestigte Flächen vorgenommen

\subsubsection{Abflusskonzentration}

Unter Abflusskonzentration wird derjenige Prozess verstanden, der die Transformation vom abflusswirksamen Niederschlag in den effektiven Gebietsabfluss beschreibt. Nachdem sich die Abflussbildung auf der Oberfläche vollzogen hat, fließt das Wasser zu Geländetiefpunkten (Einlauf, Gully) und anschließend in das Entwässerungssystem. Der Prozess der Abflusskonzentration beschreibt die zeitliche Verzögerung (Translation) und die Verformung der Ganglinie (Retention) vom abflusswirksamen Niederschlag zum Abfluss aus dem betrachteten Einzugsgebiet, wobei der Betrachtungspunkt unterschiedlich sein kann. Die Modellierung der Abflusskonzentration lässt sich nämlich sowohl zur Bestimmung des Oberflächenabflusses kleiner homogener Flächen als auch für den gesamten Gebietsausflus- 
ses größerer Einzugsgebiete verwenden, in welchen dann auch Teile des Kanalabflusses beschrieben werden. Die Größe der betrachteten Fläche kann somit um einige Größenordnungen variieren.

Generell können zwei unterschiedliche Herangehensweisen an die Modellierung dieser Effekte unterschieden werden. Dies ist a) die Berechnung nach einer Einheitsganglinie und b) die Berechnung der kinematischen Welle.

Die Einheitsganglinie beschreibt, welchen Gebietsabfluss eine Einheit abflusswirksamer Niederschlag bewirkt. Da für lineare Modelle das Superpositionsprinzip gültig ist, kann der Gebietsabfluss aus der Einheitsganglinie und dem abflusswirksamen Niederschlag bestimmt werden. Die Transferfunktion (Einheitsganglinie) kann mittels unterschiedlicher Modelle (z. B. Einzellinearspeicher, Speicherkaskade, Isochronenmethode, etc.) abgebildet werden.

Die Berechnung nach der kinematischen Welle ergibt sich aus einer Vereinfachung der Saint-Venant Gleichungen und basiert auf den physikalischen Grundprinzipien der Energie- und Massenerhaltung. In der Urbanhydrologie wird dieses Verfahren für die Berechnung des Oberflächenabflusses jedoch selten angewandt.

\subsubsection{Transportmodelle}

Für die Simulation des Abflusstransportes in den Kanalrohren sind wieder zwei unterschiedliche Konzepte mit unterschiedlicher Vereinfachung der Realität möglich. Physikalisch-mechanistische Modelle (auch hydrodynamische Modelle genannt) basieren auf den physikalischen Prinzipien der Massen- und Energieerhaltung. Konzeptuelle (hydrologische) Modelle beschreiben zwar physikalische Beziehungen, benützen aber einfache Beschreibungen der Beobachtungen.

Physikalisch-mechanistische Modelle basieren auf den Grundprinzipien der Massen- und Energieerhaltung und sind im Saint Venant Gleichungssystem beschrieben (dynamische Welle). Vereinfachungen dieser Gleichungen sind möglich und werden als diffusive Welle bzw. kinematische Welle bezeichnet. Je nach getroffenen Annahmen können somit unterschiedliche Effekte abgebildet werden (siehe Tab. 2).

Die Lösung der Saint Venant'schen Gleichungen (Typ partielle Differentialgleichungen) ist numerisch aufwendig und bedingt detaillierte Angaben zum Kanalsystem (z. B. Rohrprofil, Rauigkeit, Gefälle). Als Ergebnis der Berechnung wer-

\begin{tabular}{llll}
$\begin{array}{l}\text { TABELLE 2 } \\
\text { Berücksichtigte } \\
\text { Gleichungen }\end{array}$ & Dekte in den Vereinfachungen der Saint Venant \\
Berücksichtig & Dynamische Welle & Diffusive Welle & Kinematische Welle \\
\hline Translation & $\mathrm{Ja}$ & $\mathrm{Ja}$ & $\mathrm{Ja}$ \\
\hline Rückstau & $\mathrm{Ja}$ & $\mathrm{Ja}$ & Nein \\
\hline Dämpfung der Welle & $\mathrm{Ja}$ & $\mathrm{Ja}$ & Nein \\
\hline Abflussbeschleunigung & $\mathrm{Ja}$ & $\mathrm{Nein}$ & Nein \\
\hline
\end{tabular}

den Abflüsse und Wasserstände in den Schächten ausgegeben, wie es beispielsweise für eine Überstauberechnung nach ÖWAV-RB 112009 notwendig ist.

Konzeptionelle Strömungsmodelle basieren auf Massenbilanzen gekoppelt mit empirischen Modellen und entsprechen im Wesentlichen den Modellen die auch für die Berechnung der Abflusskonzentration eingesetzt werden (z.B. Linearspeicher, Speicherkaskade, Muskingum-Methode). Ihr Vorteil liegt in der (im Vergleich zum hydrodynamischen Modell) sehr kurzen Rechenzeit und im geringerem Datenbedarf - andererseits ist der Kalibrierungsaufwand höher. Detaillierte Daten zu Rohrprofilen sind beispielsweise nicht notwendig (De Toffol et al., 2006). Wasserstände und damit verbundene Rückstaueffekte können so allerdings nicht abgebildete werden, weshalb derartige Modelle für eine Berechnung nach ÖWAV-RB 11 2009 nicht geeignet sind. Für die Berechnungen nach ÖWAV-RB 192007 werden sie hingegen vorwiegend verwendet.

\subsubsection{Stofftransport}

Für die Modellierung der Entstehung der Verschmutzung aus den drei Quellen Trockenwetterabfluss, Niederschlag und mobilisierte Stoffe im Kanal gibt es zwei grundsätzliche Ansätze. Das erste Modell geht von einer konstanten Verschmutzung von Trockenwetter und Regenwasser aus, und berechnet die Stoffkonzentration im Mischwasser aus einer simplen Mischungsrechnung. Die Mobilisierung von Sedimenten im Kanal wird hierbei der Regenwasserverschmutzung zugeschlagen. Der zweite Ansatz berücksichtigt Akkumulation und Abtrag von Stoffen auf der Oberfläche und im Kanal und berechnet daher die Verschmutzung des Regenwassers als Funktion der Niederschlagsintensität. Obwohl das zweite Modell (auch hier sind historisch sehr unterschiedliche Formeln präsentiert worden) die physikalischen Vorgänge besser berücksichtigt, ist die Prognosequalität des Modells meist nicht signifikant besser als der simple Ansatz.
Der Transport von gelösten Stoffen in der fließenden Welle ist mit der bekannten Advektions/Dispersiongleichung zu berechnen was vor allem ein numerisches Problem darstellt. In hydrologischen Modellen kann - analog zu den vereinfachten quantitativen Ansätzen - der Transport mit Ansätzen wie z.B. Translation, Speicherkaskade, usw. simuliert werden. Zum Unterschied zu den physikalisch basierten Modellen ist das Ergebnis wesentlich von einer akkuraten Kalibrierung abhängig.

Die physikalisch basierte Berechnung des Transports von partikulären Stoffen beruht auf Sedimenttransportmodellen, die hauptsächlich im Wasserbau entwickelt wurden. Trotz langjährigen Untersuchungen und Entwicklungen werden diese Methoden aber in der Praxis nur in Ausnahmefällen eingesetzt. Im Gegensatz dazu sind empirische oder statistische Modelle für den Transport von z. B. abfiltrierbaren Stoffen weit verbreitet und in Softwareprodukten enthalten.

\subsection{Kombination von Modellen}

\subsubsection{Räumlich verteilte Modelle}

Um größere Einzugsgebiete (z. B. Kanalsysteme von ganzen Städten) berechnen zu können, muss dieses natürliche in (viele) Teileinzugsgebiete aufgeteilt werden. Die Niederschlag/Abflussberechnung erfolgt dann für jedes Teilgebiet getrennt mit unterschiedlichen Parametern für Abflussbildung und Konzentration. Die Verknüpfungen entsprechen Fließwegen im Kanalsystem, der Abfluss darin wird mittels Transportmodellen simuliert. Je detaillierter die Prozesse abgebildet werden, desto detaillierter muss auch die Aufteilung in Teileinzugsgebiete erfolgen. Während in einem hydrologischen Modell ( $A b b .3)$ die Aufteilung relativ grob erfolgt und nur die Hauptkanäle dargestellt sind, ist die Aufteilung in einem hydrodynamischen Modell (Abb. 4) wesentlich komplexer. Nur so ist es dabei möglich auch für jeden Kanalstrang die Wasserstände zu berechnen. 


\subsubsection{Integrierte Modellierung}

Integrierte Modellierung entsteht ebenfalls durch Kombination verschiedener Untermodelle für verschiedene Systeme (z. B. Kanalsysteme, Kläranlage, Wasserqualität von Flüssen, Grundwasser) in ein Gesamtmodell. Diese Kombination hat das Ziel unterschiedliche Aspekte der Siedlungsentwässerung und deren Interaktionen ganzheitlicher zu Erfassen und $\mathrm{zu}$ untersuchen (Harremoës and Rauch, 1996; Rauch et al., 1998). Beispielsweise haben so Rauch and Harremoës (1998) die Auswirkungen von Kanalnetzsteuerungen zur Verminderung von Mischwasseremissionen auf die Sauerstoffkonzentration im Fluss berechnet.

\section{Beispiele}

Am Fallbeispiel des Entwässerungssystems von Linz wird die Anwendung eines hydrologischen Modelles zum Nachweis des Wirkungsgrades der Weiterleitung nach ÖWAV-RB 19 (2007) und eines hydrodynamischen Modelles zur Beurteilung der Überstausituation gemäß ÖWAV-RB 11 (2009) aufgezeigt. Da die Datenqualität für Stadtgebiet und Umlandgemeinden unterschiedlich war, wurden die abflusswirksamen Flächen und der Trockenwetterabfluss für Stadtgebiet und Umlandgemeinden gesondert ausgewertet. Daneben wurde die hydrologische Situation für das Untersuchungsgebiet ausgewertet und bewertet. Durch eine vorab durchgeführte Sensitivitätsanalyse für verschiedene Modelleingangsparameter war bereits in der Datenerhebungsphase klar, für welche Teileinzugsgebiete die Erfassung mit größerer Genauigkeit vorgenommen werden musste. Sofern möglich wurden die Modelleingangsparameter über verschiedene voneinander unabhängige Ansätze bestimmt, um die Unsicherheiten möglichst gering zu halten. Generell wurden Messwerte den daraus abgeleiteten Größen vorgezogen. Aus diesem Grund wurde das hydrologische Modell zwar an den Entlastungsmengen kalibriert, aber der berechnete Wirkungsgrad der Weiterleitung mit den Simulationsergebnissen des hydrodynamischen Modells verglichen.

\subsection{Trockenwetterabfluss in den Teileinzugsgebieten}

Die Trockenwetterabflüsse der Umlandgemeinden wurden in 2003 in einer Messkampagne ermittelt. Die EinwohnerIn- nenzahl für die Umlandgemeinden basierte auf den Verrechnungseinheiten für die Abwassergebühren und in dem Stadtgebiet auf Daten vom Magistrat Linz (2005). Für das Stadtgebiet wurde der Trockenwetterabfluss über die Kläranlagenzulaufdaten, die für den Zeitraum von Juli 2003 bis Juni 2006 als Summenwert über 15 min vorlagen, nach ATV-DVWKA 198 (2003) bestimmt. Die Unterscheidung zwischen Regen- und Trockenwettertagen erfolgte anhand der Niederschlagsdaten der Zentralanstalt für Meteorologie und Geodynamik der Station Linz. Als Nachlaufzeit wurde unter Berücksichtigung der Ausdehnung des Entwässerungssystems eine Dauer von zwei Tagen angesetzt. Daneben wurde der Trockenwetterabfluss unabhängig von Niederschlagsdaten über das gleitende 21-Tage-Minimum nach ATV-DVWKA 198 (2003) bestimmt. Beide Methoden ergaben bis zu einem Trockenwetterabfluss von 1.500 l/s einen deckungsgleichen Verlauf und selbst die mittleren Monatswerte stimmten gut überein. Für den Trockenwetterabfluss ergab sich im Mittel ein einwohnerInnenspezifischer Wert von $280 \mathrm{~L} /(\mathrm{E} \cdot \mathrm{d})$.

\subsection{Abflusswirksame Flächen der Teileinzugsgebiete}

Für die Umlandgemeinden wurde der Anteil der befestigten, kanalisierten Fläche für verschiedene Referenzflächen auf Basis von Orthophotos abgeschätzt und soweit möglich durch Angaben des generellen Entwässerungsplans (GEP) von 2005 ergänzt. Diese Versiegelungsgrade wurden auf die restlichen Teilflächen der Umlandgemeinde hochgerechnet. Fehlende Daten wurden aufgrund der Flächencharakteristik abgeschätzt. Im Stadtgebiet ergab sich die kanalisierte Fläche aus den Verkehrsflächen, den als Bauland ausgewiesenen Flächen und den Sonderflächen laut Magistrat Linz (2005). Ebenso war die befestigte, kanalisierte Fläche größtenteils bekannt. Die Flächen eines Stadtbezirks wurden jeweils zur Gänze einem Hauptsammler zugeordnet.

\subsection{Hydrologische Situation im Raum Linz}

Die einzige Niederschlagsreihe mit hinreichend langer Dauer für statistische Auswertungen nach ATV-A 121 (1985) lag außerhalb des Einzugsgebietes in Wels. Ein Vergleich von 43 Einzelereignissen der Starkregenserie der Jahre 1993 bis 2004 von Linz-Stadt mit Wels ergab eine starke Abweichung der Spitzen bei guter Übereinstimmung der Regensummen. In der kontinuierlichen Simulation führten vier im Einzugsgebiet verteilte Regenschreiber im Vergleich zur Regenserie der Station Linz-Stadt zu einer Reduzierung des Überstaus der Schächte, der Überstaudauer und des Gesamtentlastungsvolumens. Trotz dieses Simulationsergebnisses wurde die Regenreihe der Station LinzStadt nicht abgemindert, da die Regenreihe von Linz-Stadt geringere Intensitäten als die übrigen Stationen aufwies.

\subsection{Mischwasserentlastungsmengen und Entlastungsereignisse}

Der Zufluss zur biologischen Stufe der Regionalkläranlage wird hauptsächlich über fünf Mischwasserentlastungsbauwerke reguliert. Aus Messungen des Wasserstands in Entlastungsbauwerken lassen sich keine exakten Aussagen über die tatsächlichen Entlastungsvolumina gewinnen, die aber für die Kalibrierung eines hydrologischen Modells erforderlich sind (Sitzenfrei et al., 2008). Vielmehr lässt sich nur die Anzahl der Entlastungsereignisse durch Vergleich der Wasserstandshöhe im Kanal mit der zugehörigen Höhe der Überlaufschwelle bestimmen. Für das Entlastungsbauwerk nach dem Vorklärbecken wurde das Entlastungsvolumen für Juli 2003 bis Juni 2006 aus der Differenz von $\mathrm{Zu}$ - und Abflussmengen quartalsweise abgeschätzt.

\subsection{Aufbau und Kalibrierung des hydrologischen Modells}

Die hydrologischen Simulationsberechnungen wurden mit dem Programm CITY DRAIN (Achleitner et al., 2007) in der Version 2.0 durchgeführt, in dem die wesentlichen Elemente eines Entwässerungssystems als Blöcke eingegeben werden. Die Abflussbildung erfolgt über einen einfachen Verlustansatz unter Berücksichtigung von Anfangs- und Dauerverlusten und der Abflusstransport über eine modifizierte Form der Muskingum-Gleichung. Die Fließzeit beruht auf der Annahme einer Dreiviertelfüllung des maßgeblichen Hauptsammlers im Einzugsgebiet. Die Berechnungsschrittlänge ist durch die zeitliche Auflösung der Niederschlagsdaten von fünf Minuten vorgegeben.

Um im Modell einen einheitlichen Grad der Detailliertheit für die Teileinzugsgebiete zu erreichen, wurden mehrere reale, nicht durch Pump- oder Entlastungsbauwerke getrennte Teileinzugsgebiete mit 


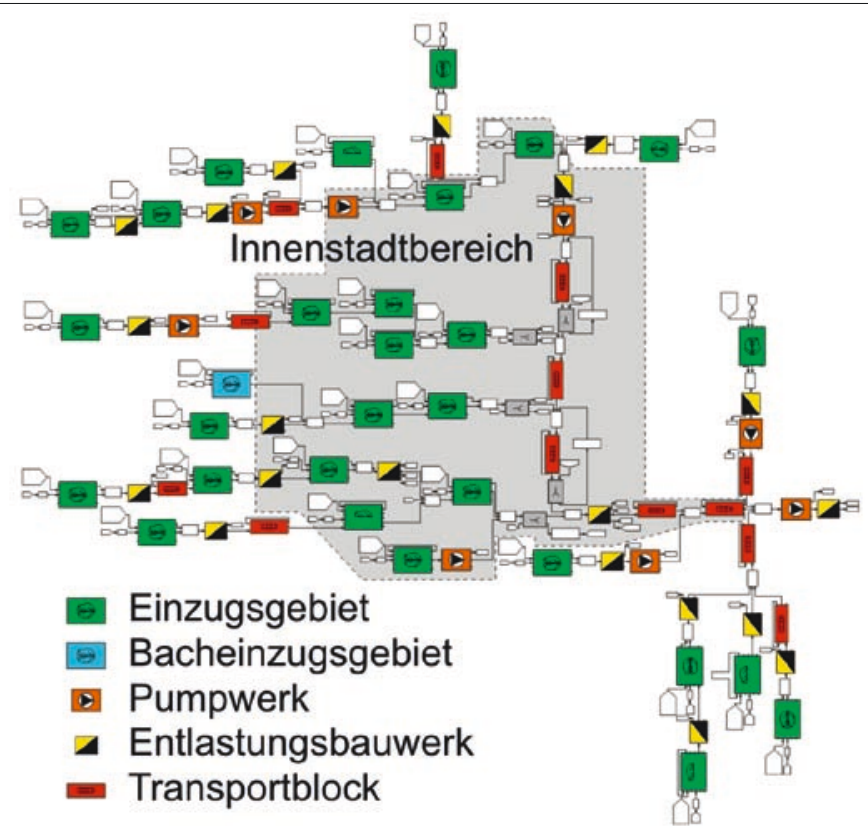

Abb. 3: Hydrologisches Modell des Entwässerungssystems von Linz.

ähnlichen Fließzeiten zu einem Einzugsgebiet zusammengefasst. Die im Kanalsystem wirksame Abflussretention wurde über die Angabe eines zusätzlichen Retentionsvolumens erfasst. Das Modell beinhaltete alle relevanten Hauptsammler zwischen den inneren Umlandgemeinden und der Stadt sowie im Stadtgebiet parallel zur Donau. Bei Entlastungsbauwerken mit fehlenden Angaben zu Volumen oder Drosselabfluss wurden die nicht vorhandenen Werte von Bauwerken mit geringem Beitrag zum Gesamtsystemverhalten ingenieurmäßig berechnet.

In hydrologischen Modellen ist die Umsetzung einer Steuerung mit einem erhöhten Abstraktionsaufwand verbunden, da Wasserstände nicht direkt als Steuerungssignale verwendet werden können. Eine genaue Beschreibung zur Umsetzung der zweistufigen Weikerlsee-Steuerung zur Verhinderung einer Entlastung in den Weikerlsee findet sich in Kleidorfer et al. (2007).

Die Kalibrierung des hydrologischen Modells erfolgte mittels Kontinuums-Simulation für die Jahre 2003 und 2005 anhand der in Abbildung 3 dargestellten Entlastungsbauwerke über die Anzahl und das Volumen der Entlastungsereignisse. Da im Winter aufgrund von Schneefall und Abschmelzvorgängen auf der Oberfläche nur selten ein direkter Zusammenhang zwischen Niederschlag und Abfluss hergestellt werden kann, wurde für die Kalibrierung ausschließlich der Zeitraum von April bis Oktober betrachtet.
Für die Kalibrierung wurden die simulierten Entlastungsvolumina den gemessenen gegenübergestellt. Im Zuge der Kalibrierung wurden in den innerstädtischen Bezirken $30 \mathrm{~m}^{3} / \mathrm{ha}_{\mathrm{E}, \mathrm{b}}$ und in den Umlandgemeinden $10 \mathrm{~m}^{3} / \mathrm{ha}_{\mathrm{E}, \mathrm{b}}$ als aktivierbares Kanalspeichervolumen angesetzt. Anschließend wurde die abflusswirksame
Fläche der Einzugsgebiete schrittweise um je $10 \%$ reduziert bis eine ausreichende Übereinstimmung der simulierten mit den gemessenen Entlastungsvolumina erzielt wurde.

\subsection{Aufbau und Kalibrierung des hydrodynamischen Modells}

Für die hydrodynamischen Berechnungen wurde die Simulationssoftware MIKE URBAN 2005 verwendet. Zur Reduzierung der Rechenzeit wurde mit dem in Abbildung 4 gezeigten Grobmodell gearbeitet. Damit lassen sich aus den Simulationsergebnissen zwar keine gesicherten Rückschlüsse hinsichtlich der realen Überstausituation ableiten, aber zumindest mögliche Problembereiche ermitteln, die dann in einem fein aufgelösten Teilmodell näher zu untersuchen sind. Die Umlandgemeinden sind jeweils mit einem Einzugsgebiet abgebildet. Um im innerstädtischen Bereich überstaugefährdete Stellen möglichst gut zu erfassen, wurde jeder Stadtbezirk soweit als möglich als eigenes Einzugsgebiet abgebildet. Die Abmessungen wichtiger Entlastungs- und Pumpbauwerke beruhen auf der Auswertung von Plänen. Düker wurden jeweils durch eine Haltung des gleichen Durchmessers ersetzt.

Für die Abflussbildung wurde ein auf dem Linearspeicheransatz basierendes

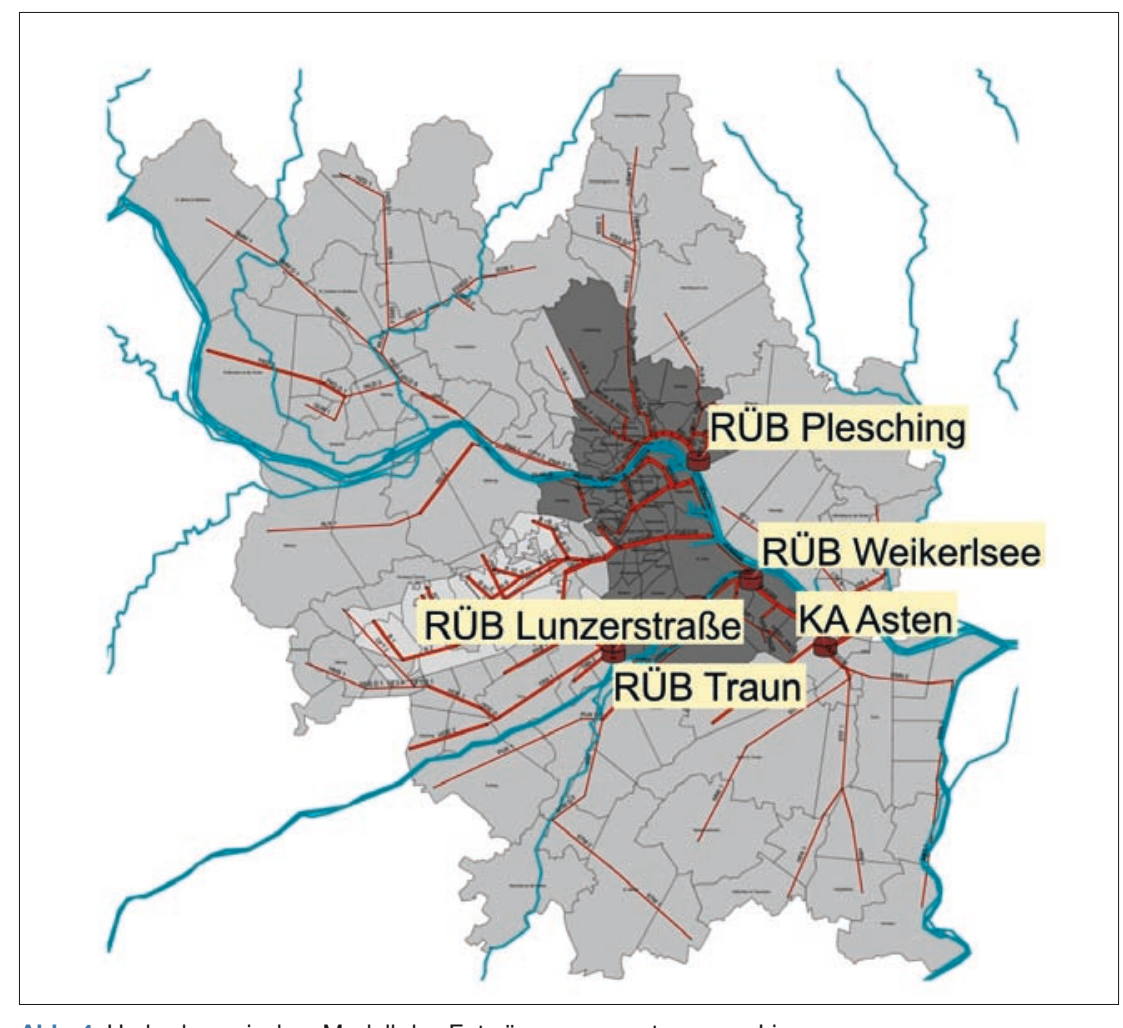

Abb. 4: Hydrodynamisches Modell des Entwässerungssystems von Linz. 


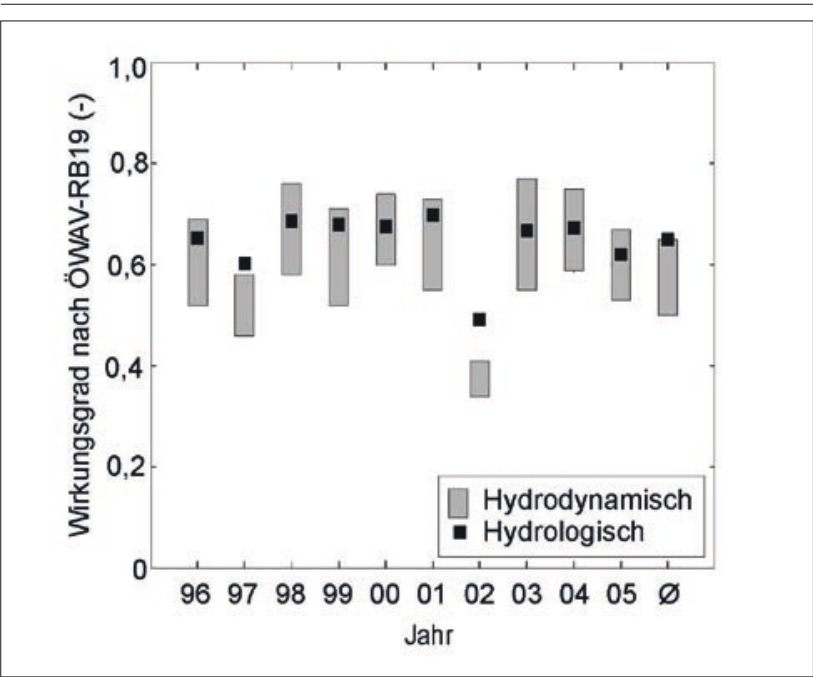

Abb. 5: Wirkungsgradvergleich von hydrodynamischer und hydrologischer Simulation.

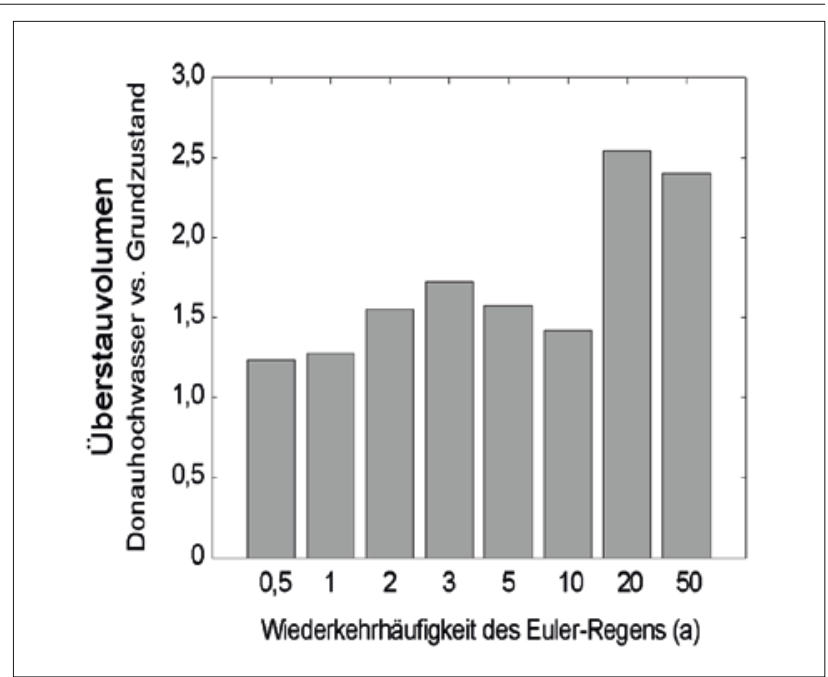

Abb. 7: Abhängigkeit des Überstauvolumens von der Wiederkehrhäufigkeit.
Modell gewählt, in dem Anfangsverluste, Evaporation und Infiltrationsverluste nach der Gleichung von Horton berücksichtigt werden. Die Evapotranspiration ging aufgrund fehlender Temperaturund Windgeschwindigkeitsdaten für die Trockenperiode stark vereinfacht in das Modell ein. Die gebietsspezifische Speicherkonstante resultierte aus der Fließgeschwindigkeit im Hauptsammler und der Größe der befestigten Fläche. Für die Abflussbildung wurde im Modell eine Einheitsganglinie unter Anwendung des Prinzips der linearen Speicherkaskade definiert. Die Schachtdurchmesser wur- den nur soweit wie erforderlich in das Modell übernommen, ansonsten wurde ein Schachtdurchmesser von $1 \mathrm{~m}$ angesetzt.

Auch im hydrodynamischen Modell wurde die Weikerlsee-Steuerung in einer zweistufigen Ausführung implementiert, allerdings wurde die reale zeitversetzte Schließung der vier Schieber durch unterschiedliche Schließgeschwindigkeiten simuliert.

Das hydrodynamische Modell wurde an sechs ausgewählten Regenereignissen aus dem Zeitraum Juni 2004 bis Juli 2005 kalibriert wobei die Regenereignisse un-

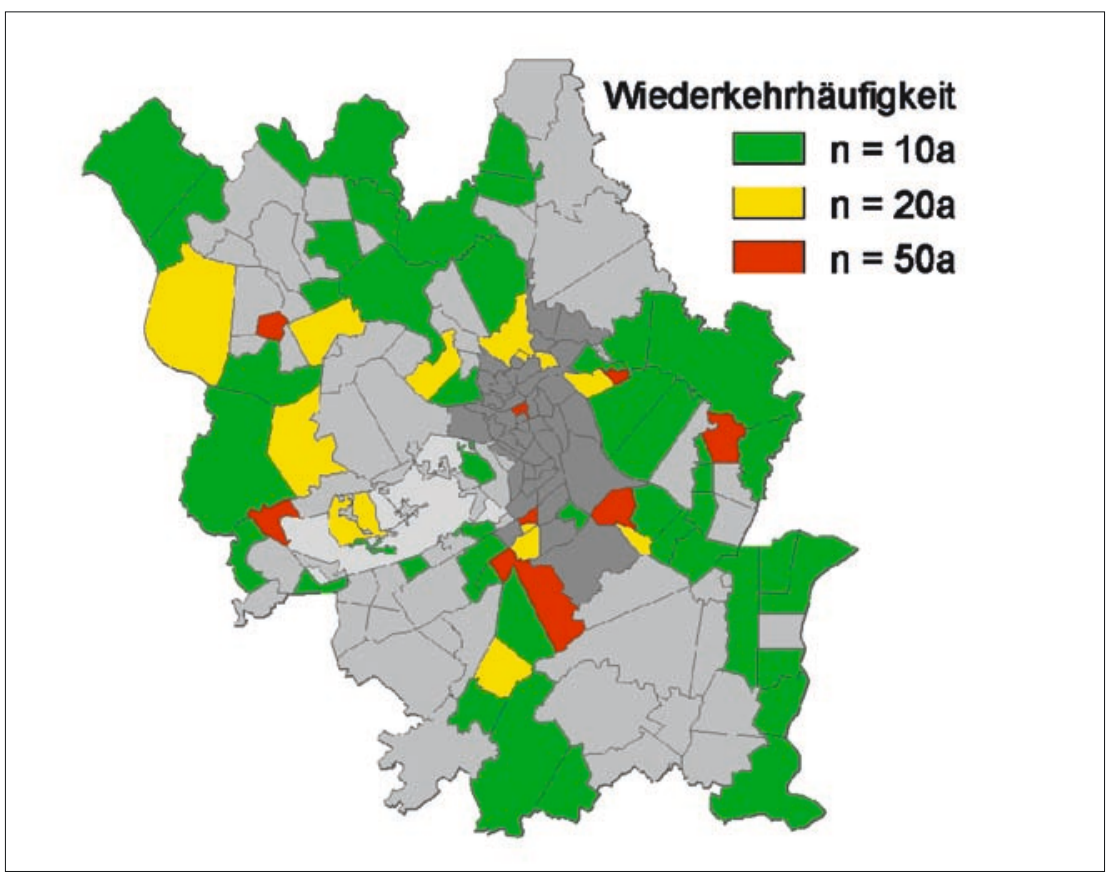

Abb. 6: Ergebnisse des Überstaunachweis mit Modellregen unterschiedlicher Wiederkehrhäufigkeit. terschiedlichen Wiederkehrhäufigkeiten entsprachen, d. h. unterschiedliche Intensitäten auswiesen.

\subsection{Wirkungsgrad der Weiterleitung gemäß Regelblatt 19}

Der Wirkungsgrad der Weiterleitung nach ÖWAV-RB 19 (2007) wurde mittels Langzeit-Kontinuums-Simulation über 10 Jahre mit beiden Modellen berechnet. Da sich der Wirkungsgrad der Weiterleitung über die Entlastungsmenge oder über die Kläranlagenzulaufmenge und den Oberflächenabfluss berechnen lässt, führen unvermeidbare Volumenfehler in der hydrodynamischen Simulation dazu, dass die nach der Theorie identischen Werte in der Praxis variieren. Von Bedeutung ist hier die durch die iterative numerische Lösung der Differentialgleichungen zustande kommende Volumendifferenz, die im Anwendungsbeispiel bei ca. 1,5\% des Gesamtabflussvolumens liegt und damit ca. $40 \%$ des Entlastungsvolumens entspricht. Die obere bzw. die untere Grenze des Ergebnisintervalls ergibt sich durch die jahrweise Beaufschlagung der Volumendifferenz auf das Kläranlagenzulaufvolumen bzw. auf das Entlastungsvolumen. In der Realität ist der Wirkungsgrad der Weiterleitung irgendwo innerhalb des Ergebnisintervalls zu erwarten.

In Abbildung 5 sind die mit den beiden Modellen berechneten Wirkungsgrade jahrweise gegenübergestellt. Die Simulationsergebnisse des hydrologischen Modells liegen größtenteils im Ergebnisintervall des hydrodynamischen Modells, so dass die beiden Modelle gut übereinstimmen. Nur für die regenreichen Jahre 1997 
und 2002 mit sehr starken Niederschlagsereignissen bewirken die unterschiedlichen Ansätze für die Abflussbildung, dass das hydrologische Modell höhere Wirkungsgrade liefert als das hydrodynamische Modell.

\section{8. Überstaunachweis gemäß Regelblatt 11}

In ÖWAV-RB 11 (2005) ist als Schutz vor Überflutung die zulässige Wiederkehrhäufigkeit in Abhängigkeit der Gebietsnutzung festgelegt. Der sich einstellende Überstau wurde für Modellregen vom Typ Euler II und eine Starkregenserie berechnet und schachtweise sowie gebietsweise ausgewertet. Die Simulationsergebnisse lassen beim schachtspezifischen Überstauvolumen aufgrund des verwendeten Grobmodells keine gesicherten Rückschlüsse auf die reale Überstausituation $\mathrm{zu}$. So ließen sich Überstaueffekte bei den Anfangsschächten der Umlandgemeinden durch die konzentrierte Einleitung aufgrund der gröberen Netzauflösung erklären.

Aus der Simulation mit Euler II-Modellregen gingen für eine Wiederkehrhäufigkeit von fünf Jahren drei überstaugefährdete Bezirke für das Stadtgebiet hervor. In den Umlandgemeinden führte der zweijährige Modellregen vom Typ Euler II bei acht Einzugsgebieten zu Überstau. In Abbildung 6 ist die gebietsweise Auswer- tung für die Berechnungen mit zehn- bis fünfzigjährigem Modellregen dargestellt.

Bei der Simulation mit der Starkregenserie wurden die maximalen Wasserstandshöhen statistisch ausgewertet. Generell konnten durch die Simulation mit der Starkregenserie die Ergebnisse mit Modellregen vom Typ Euler II bestätigt werden. Während im Stadtgebiet die überstaugefährdeten Gebiete identisch mit der Simulation mit Modellregen waren, nahm bei den Umlandgemeinden die Anzahl der überstaugefährdeten $\mathrm{Ge}$ biete leicht zu.

Aus Abbildung 7 wird deutlich, dass ab einer Wiederkehrhäufigkeit von 20 Jahren das Überstauvolumen signifikant zunimmt und damit von einer Überlastung des Entwässerungssystems auszugehen ist. Da mit größeren Wiederkehrhäufigkeiten die Zahl der Schächte mit Überstau nur gering ansteigt, muss das zusätzliche Überstauvolumen örtlich konzentriert an den relevanten Entlastungsbauwerken anfallen.

\section{Zusammenfassung und Ausblick}

Die Entwicklung numerischer Modelle zur Berechnung des Verhaltens von Entwässerungsanlagen änderte die Möglichkeiten in der Dimensionierung von Anlagen. Waren bislang Bemessungsformeln und empirische Beziehungen vorherrschend, so stehen heute komplexe Software Werkzeuge zur Verfügung. In Österreich wurde den geänderten Bedingungen kürzlich innerhalb der Regelwerke Rechnung getragen. Sowohl für die Dimensionierung von Kanälen als auch für die Bemessung von Mischwasserentlastungen wurde der Einsatz von numerischen Modellen zum Stand der Technik erklärt. Die Anwendung der Software stellt im Vergleich zur bisherigen „Papier und Bleistift“ Berechnung sicher einen höheren Aufwand dar, insbesondere durch den Aufwand der Kalibrierung der Modelle. Dem steht aber ein sehr hoher Erkenntnisgewinn gegenüber, wie hier anhand des Beispiels Linz dargestellt wurde. Nur durch den Einsatz moderner numerischer Methoden ist es möglich fallspezifisch optimierte Lösungen zu finden.

\section{Danksagung}

Die Autoren bedanken sich bei der LinzAG für die gute Zusammenarbeit und die finanzielle Förderung im Rahmen eines Projekts.

Korrespondenz:

Univ. Prof.Dr. Dr. Wolfgang Rauch

DI. Dr. Manfred Kleidorfer

Dr.-Ing. Stefan Fach

Arbeitsbereich Umwelttechnik

Universität Innsbruck

Technikerstrasse 13

6020 Innsbruck

E-Mail: wolfgang.rauch@uibk.ac.at

\section{LITERATUR}

2000/60/EC (2000) Directive of the European Parliament and of the Council establishing a framework for Community action in the field of water policy

Achleitner S, Moderl M, Rauch W (2007) CITY DRAIN (C) - An open source approach for simulation of integrated urban drainage systems. In: Environmental Modelling and Software, Vol. 22(8), p. 1184-1195

ATV-A 121 (1985) Niederschlag - Starkregenauswertung nach Wiederkehrzeit und Dauer, Niederschlagsmessungen, Auswertung. ATV e.V., Hennef

ATV-DVWK-A 198 (2003) Vereinheitlichung und Herleitung von Bemessungswerten für Abwasseranlagen. ATV-DVWK e.V., Hennef De Toffol S, Kleidorfer M, Rauch W (2006) „Vergleich hydrodynamischer und hydrologischer Simulationsmodelle bei der Berechnung der Emissionen von Mischwasserbehandlungsanlagen." Wiener Mitteilungen 196:

\section{$\mathrm{H} 1-\mathrm{H} 20$}

Harremoës P, Rauch W (1996) „Integrated design and analysis of drainage systems, including sewers, treatment plant and receiving waters." Journal of Hydraulic Research 34(6): 815-826 Johansen NB (1985) Discharge to receiving waters from sewer systems during rain. PhD thesis. Technical University of Denmark

Technical University of Denmark
Kleidorfer M, Fach S, Möderl M, Rauch W (2007) Umsetzung von Kanalnetzsteuerungen in hydrologischen Modellen am Beispiel von Linz. In: Österreichische Wasser- und Abfallwirtschaft,
Vol. 59(9-10), p. 131-137

Kuichling E (1889) The relation between the rainfall and the discharge of sewers in populous districts. Transactions of the American Society of Civil Engineers, 20, 1-60

Magistrat Linz (2005) Linz Zahlen - statistische

Bezirke. Stand: Juli 2006, http://www.linz.at/ Bezirke. Stand: Juli 2006, http

Möderl M, Kleidorfer M, Fach S, Rauch W (2007) Reduktion von Mischwasseremissionen durch Optimierung eines Entwässerungssystems am Beispiel der Stadt Linz. In: Wiener Mitteilungen, Vol. 203, p. K1-K22

Mulvaney TJ (1851) On the use of self-registering rain and flood gages, in making observations of the relation of rainfall and flood discharges in a given catchment. Proceedings of the Institute of Civil Engineers of Ireland, 4, 18-31 ÖNORM EN 752 (1997) Entwässerungssysteme außerhalb von Gebäuden

ÖWAV-RB 11 (2005) Richtlinie für die abwassertechnische Berechnung und Dimensionierung von Abwasserkanälen (in Bearbeitung). Österreichisches Normungsinstitut, Wien

ÖWAV-RB 11 (2009) Richtlinie für die abwassertechnische Berechnung und Dimensionierung von Abwasserkanälen; 2. vollständig überarbeitete Auflage, Österreichisches Normungsinstitut, tete Wien

ÖWAV-RB 19 (2007) Richtlinie für die Bemessung von Mischwasserentlastungen. Österreichischer Wasser- und Abfallwirtschaftsverband, Wien
Paulsen O (1987) Kontinuierliche Simulation von Abflüssen und Stofffrachten in der Trennentwässerung. Universität Hannover, Heft 62 Rauch und Kinzel (2007) KAREN Manual. hydro-IT Innsbruck

Rauch W, Krejci V, Frutiger A, Gujer W (2001)

Generelle Entwässerungsplanung in der Schweiz - REBEKA und der neue Zustandsbericht Gewässer. Korrespondenz Abwasser. (48) No 11, pp. $1615-1622$

Rauch W, Harremoës P (1998) "Correlation of combined sewer overflow reduction due to realtime control and resulting effect on the oxygen concentration in the river." Water Science and concentration in the river."
Technology 37(12): 69-76

Rauch W, Aalderink H, Krebs P, Schilling W, Vanrolleghem P (1998) „Requirements for integrated wastewater models - Driven by receiving water objectives." Water Science and Technology 38(11): 97-104

Rauch W, Thurner N, Mikkelsen PS, Stegner U (1998) Infiltration of Urban Runoff under Consideration of the Joint Probability of Rain and High Groundwater Levels. In: Proceedings of the 3rd international conference on innovative technologies in urban storm drainage. NOVATECH 1998, Lyon, France

Sitzenfrei R, Fach S, Rauch W (2008) Auswirkungen von Vereinfachungen bei der Bestimmung von Mischwasserentlastungsmengen quo vadis Poleni? In: Wiener Mitteilungen, Vol. 209, p. H1-K23 